Relativistic Quantum Mechanics : Wave Equations (Theoretical Physics-Text and Exercise Books, Vol 3)

by W Greiner

Springer-Verlag: Berlin-Heidelberg-New York-London-Paris-Tokyo, Hong Kong, 1990 xvi +345 pages, 62 figures ; price : DM 75 (Soft cover) ; ISBN 3-540-50986-0

In this book the author deals mostly with Dirac's relativistic wave equation. Out of a total of 338 pages 193 pages are devoted to the Dirac equation and the rest to the Klein-Gordon equation, Weyl's neutrino equation, wave equations for arbitrary spin and the Lorentz group. The only other book containing a comprehensive treatment of the Dirac equation is M E Rose's Relativistic Electron Theory (John Wiley 1961). But, the two books differ widely in content and presentation. While Rose Deals mostly with the theoretical aspects of the equation Greiner concentrates on its applications. The book fills a gap in the literature on the subject. The numerous worked put problems will give the student a deep insight into relativistic quantum mechanics.

Chapter 1 of the book contains a detailed treatment of tha Klein-Gordon (KG) equation for particles of spin zero. This topic is sketchily discussed in most of the other books. The equation is set up and the expressions for the charge-current densities for the free particle are derived. From the KG equation Schrodinger's equation is obtained in the nonrelativistic limit by splitting the time dependence of $\psi$ into two parts and separating the rest-mass dependent part $\exp \left(-\frac{i}{\hbar} m_{0} c^{2} t\right)$.

Next, it is shown how to obtain the wave equations and the energymomentum tensor from a general Lagrangian density, $\alpha\left(\psi_{\sigma}, \partial \psi_{\sigma} / \partial x^{\mu}\right)$. The KG equation and the Schrödinger equation and the corresponding energy-momentum tensors are then obtained as special cases.

The invariance properties of the KG equation are discussed and its Lorentz invariance is established. One of the uncommon topics discussed in the book is the Feshbach-Villars representation, the so-called $\phi$ representation, in which the prsitive and negative charge solutions are always of the form

$$
\phi^{+}(p),\left(\begin{array}{l}
1 \\
0
\end{array}\right), \phi^{-}(p) \sim\left(\begin{array}{l}
0 \\
1
\end{array}\right)
$$


The Lagrange density in the $\phi$ representation is constructed and from it the energy-momentum tensor and the Hamiltonian are derived. The free particle solutions in the $\phi$ representation are found to be of the expected type $\left(\begin{array}{l}1 \\ 0\end{array}\right),\left(\begin{array}{l}0 \\ 1\end{array}\right)$, for positive and negative charges.

In Section 1.9 the interaction of a spin-0 particle with and electromagnetic field is introduced in the usual way by changing $p_{\mu}$ to $p_{\mu}-\frac{e}{c} A_{\mu}$. In this case the expressions for the charge-current densities are found to involve the potentials. In Section 1.10 the KG equation for a central field is separated into radial angular parts. The radial equation for a Coulomb field is solved in the next Section and the solution used to determine the energy levels of the pionic atom with point-like nucleus. The equation is then solved for the potential of a homogeneously charged sphere, the square well and an exponential potential, and diagrams are drawn to illustrate the results. In Sections 1.18, 1.19 the author returns to the $\phi$ representation and calculatos the position operator and the current density for particles and antiparticles. The last two Sections of Chapter 1 are devoted to a discussion of the Kisslinger potential and the Lorentz-Lorentz type effect in pion-nucleon scattering with which the student is not likely to be familiar.

Chapters 2 to 13 (pp. 75-267) are devoted to discussions on the Dirac equation with emphasis on applications. As the Chapters 2 to 8 do not contain anything that cannot be found in other books, these Chapters can be passed over with only a reference to Section 8.5 where the time evolution of a Gaussian wave packet composed of positive and negative energy states is studied. In Chapter 9 is considered the problem of determining the eigenvalues and eigenfunctions of a one-dimensional square-well potential. In the corresponding nonrelativistic problem two conditions are to be satisfied at each boundary of the well, one for matching the values of the functions and the other for matching the derivatives. In the relativistic case the continuity of the current requires that only the values of the functions on the two sides of a boundary need be matched, and this, apparently, can give only one condition to be satisfied by the amplitudes. The situation is clarified by treating the spin-up and spin-down cases separately. In the spin-up cas e, for instance, the nonvanishing components of the Dirac $\psi$ are $\psi_{2}$ and $\psi_{3}$. Matching these two functions across a boundary imposes two conditions on the amplitudes of the waves travelling in opposite directions along the z-axis. Thus, from the mathematical standpoint thrre is no essential difference between the relativistic and the nonrelativistic problem. The same technique can be used for determining the transmission coefficients of an infinite potential step and of a rectangular potential barrier. Essentially the same technique is used for solving the problems of (i) onedimensional potential well with scalar coupling, (ii) a spherical potential box with $V=-V_{0}$ for $r \leqslant R$ and $V=0$ for $r>R$, (iii) the hydrogen atom and (iv) a Dirac particle in a Coulomb and a scalar potential. These worked out problems will 
enable the student to construct solutions of the Dirac equation by joining solutions corresponding to different potentials on the two sides of a boundary. Such problems often arise in atomic, nuclear and elementary particle physics.

In Chapter 10 the two-centre Dirac equation is taken up for discussion. Exact solutions of the nonrelativistic problem were given independently by $E$ Teller (1930), E A Hylleraas (1931) and G Jaffé (1934) and applied to molecular problems. But, the numerical calculations were laborious and often needed the use of computers. The relativistic problem was solved for the first time by $B$ Müller and W Greiner in 1973 (Phys. Lett. 47B, 5(1973) ; Z. Naturf. 31a, 1(1976)). The solution was obtained in prolate spheroidal coordinates $\xi, \eta$ in series of products of Laguerre polynomials in $\xi$ and Legendre polynomials in $\eta$. This and the HartreeFock solution of the problem given by the same authors from the subject matter of this Chapter.

In Chapter 11 the Foldy-Wouthuysen transformation both in the presence and in the absence of a field is discussed at considerable length. This topic is also discussed in other books. In Chapter 13 Klein's paradox that an electron can mount a potential barrier of height greater than its kinetic energy by changing the sign of its formulated both by Klein's original method (Z. Phys. 53, 157 (1929)) and by the method of Chapter 9 , Section 1.

After discussing Weyl's two-component neutrino theory the author proceeds to set up first order wave equations for particles with arbitrary spin $s=1, \frac{1}{2}, 2 \ldots$ in Chapter 15. The multispinors representing such particles are symmetrized tensor products of zero momentum solutions, $\omega_{\alpha}^{(r)}(0)=\delta_{r \alpha}$, of the Dirac equation. From the general theory the Bargmann-Wigner, Proca and Duffin-Kemmer equations are obtained as special cases. Spin $\frac{3}{2}$ fields are discussed in Section 15.6 and the Rarita-Schwinger and the Fierz-Pauli-Gupta equations are derived.

The last Chapter contains discussions on the Lorentz group $0(3,1), 4$-dimensional rotation group $0(4)$, the 3 -dimensional rotation group $0(3)$, the group $S L$ $(2, \mathrm{C})$ of unimodular 2-dimensional complex matrices and the group SU(2). The inhomogeneous Lorentz group and the conformal group are also discussed briefly. The discussions, though elementary, will be useful to students with little or no group-theoretical background. The generators are found and the commutation relations satisfied by them written down. The two-to-one homomorphism between the proper Lorentz group $L_{p}$ and the group $S L(2, C)$ and between $0(3)$ and $S U(2)$ is established. The homomorphism is then used to find the irreducible representations of $O(3)$ and $L_{p}$ as is done in other books. The connection between spatial rotation and spin is also discussed. 


\title{
Shifting Cultivation in India
}

\author{
by Sachchidananda
}

Concept Publishing Company : New Delhi 110 059, 1989

xiv + 181 pages ; price : Rs. $100.00 / \$ 20$; ISBN 81-7022-040-8

The book under review puts forward a very important issue relating to a particular means of subsistence practised by a large section of the people. The very pattern of cultivation centering round the rotation of land and the firing of the cut down shrubs and very often tress, known as shifting or swidden cultivation, is still widespread in the different parts of the world. This process does not need plough to turn over the soil and in this the use of human labour is the main output. The shifting cultivation has aroused many questions as regards its destructive nature despite its overall utilization in the production of crops by the considerable section of the people. As an uneconomic and wasteful means of cultivation it has had to face severe criticism and in the interest of the forest resources and improved control of land it has been decided by many countries of the world to stabilize mobile agricultural system characterised by swidden cultivation.

The swidden cultivation, in most of the tribal groups, forms the basic substratum of economic life. If reflects the interacting patterns of environment and culture. This process of cultivation has a very long root into the socio-economic settings of many population groups. Sometimes these are so integrated into the basic philosophy of the people that it demands a very deep oriented study into the system to understand the impact of this cultivation pattern on the social groups.

The very intention of the book in question is practically centred round this attitude of study. The swiddeners throughout the country do not all alike and they do not necessarily share the same view-points and common philosophy. Thus there is a basic need for close-set studies on the different regions and on different communities. Moreover, there is a specific need for understanding the nature and extent of the swidden cultivations practised in the major regions on the globe and to evaluate the interactions of environment and culture among these diversified human groups with a view to illustrate the global trend on the swidden cultivation. With this end in view a major project of research was taken up under the auspices of UNESCO's Man and Bio-sphere (MAB) programme to conduct a cross cultural study on shifting cultivation practised in five countries in Asian region viz. India, Indonesia, Malayasia, the Philippins and Thailand. The author of the book, Professor Sachchidananda, was given the responsibility by the concerned authorities to prepare the country profile for India on that research issue. The materials thus colfected were used in the book, published by the UNESCO, entitled Swidden Cultivation in Asia. The present book is the outcome of the utilization of the material collected and evaluated in Indian context. 
Professor Sachchidananda deserves special credit in the systematic presentation of a broad-based perspective of the swidden cultivation and in so doing he had left no stone unturned to acquaint the readers with the basic principles of this cultivation derived from the swiddeners throughout the world. The swidden cultivation in India has been assessed and analysed in the background of social geography, ecology, social diversity and religious sentiments of the country. The man-land relationship in the different regional centres of shifting cultivation is perfectly illustrated and understood through the integrated perspective of ecology and culture of the people concerned.

There has been a number of anthropological studies on shifting cultivation and these are mostly based on highlighting the traditional ethnographic dimension and the cultural barriers that led to many narrow-ended situations. No attention has keen paid by these scholars to the changes in the traditional life-ways and thoughtways resulted from various external factors. After independence rapid process of implementation of various developmental factors has changed the whole situation. The growing demand for land, the increasing prices of timber have jointly thrown the swiddeners in specific troubles in getting requisite lands for cultivation. With these have added the new waves of change in consequence of various developmental measures taken up by the government.

It has been observed that the existence of very strongly based cultural imperatives in the background of swidden cultivation resists the motion for stoppage or change if in any such community, practising this sort of cultivation. But the author believes that it is not wholly correct in all the case. There are changes in the life of the swiddeners despite conspicuous socio-cultural constraints. The change, as the author believes, is not completely absent, rather there are many swidden practising communities who have accepted some changing situations under the pressure of diverse factors. According to the author, the relocation scheme for some shifting cultivators, in the second Five Year Plan period, could not bring any success not due to the cultural barriers and strong social taboos but "on account of faulty administrative arrangements" regarding the making of adequate preparation for the change.

Shifting cultivation has developed a complicated life-pattern since long time past and thus it should not be assessed superficially. It is a direct response to demands of an eco-system and in it there is an integrated attempt of adjustment with a particular environment. As the human factors are directly involved in these attempts, so there are conspicuous cultural impacts conditioned by value-attitude system. Thus, any efforts to explore this cultivation system must be associated with a trend of inter-disciplinary investigation pattern and specific area-based survey work.

The book is broadly divided into two parts. Part -1 explores the country profile of the shifting cultivation i.e. it is based on the study and analysis of this 
particular cultivation in an all India level. This situation has been examined through a case study in Arunachal Pradesh, which, according to the author, is the "traditional home of the swiddeners". Through a broad-based study on the Wancho-a primitive tribe, living in five villages viz. Pangchaw, Niausa, Rusa, Mintong and Zedua in Arunachal Pradesh - the author has presented an effective all-purpose study on the swidden cultivation and the Swiddeners in a particular region. It speaks many things relatirig to the social, historical and environmental perspectives of the tribe-a traditional shifting cultivator group-and their multi-dimensional interaction with totalistic pattern of life. Since all economic relations are set in a social framework, so it is desirable, according to the author, to evaluate each case of swiddening in its specific historical, physiographic and socio-political context. It is very interesting to note that after a broad-based study on the swidden cultivation practised by the Wancho the author does not consider the swidden as uneconomic and in this line he differs from the existing and widespread concept. He advocates for a positive policy towards shifting cultivation and thus he suggests for modification of the system what he prefers to call scientific swiddening. But it is hard to believe that through the improvement of the patterns of such cultivation the large-scale and deep-based injury to the natural surroundings could be saved. The jhuming is basically related to the felling of trees and shrubs, and if it goes on unabated, how can we be able to check netural imbalance which is threatening us constantly?

The book is well-written and the layout of different materials is highly praiseworthy. It, no doubt, provides a guide line for studying other swiddeners in the country.

R M S ARKAR Department of Anthropology, Bangabasi College, 19 Scott Lane, Calcutta-700009

Recent Developments in Surface Acoustic Waves (Springer Series in Wave Phenomena, Vol 7)

(Proceedings of European Mechanics Colloquium 226, University of Nottinghum, U.K., September 2-5, 1987)

edited by D F Parker and G A Maugin

Springer-Verlag: Berlin-Heidelberg-New York-London-Paris-Tokyo-Hong Kong , 1988 $x+351$ pages, 139 figures ; price: DM 110 (Hard cover) ; ISBN 3-540-19401-0

This book is based on the papers presented at the European Mechanics Colloquium 226 held at the University of Nottinghum, UK, during September 2-5, 1987 and deals with surface acoustic waves. The subject of surface waves is multidisci- 
plinary and lies at the interface between Physics, theoretical and applied Mechnics, electroacoustics, applied Mathematics, Surface Science, elasticity and seismology. Although the papers show diversity in approach and background, they also exhibit strong links between the phenomena arising from different fields. The papers highlight recent developments such as nonlinear and nonclassical effects and these have important applications to signal processing, nondestructive testing and seismic studies.

Part I of this book deals with the recent advances in the mathematical treatment of nonlinear effects, of viscoelastic and of some new constitutive effects which modify the predictions of linear elastic and piezoelectric theory for propagation of surface acoustic waves (SAW).

Part II is devoted to the study of surface waves on anisotropic substrates. In particular the behaviour of surface waves in transversely isotropic elastic media is investigated in detail and a new method is presented for calculation of surface impedance.

Part III is concerned with the analysis of surface waves travelling across rough or corrugated surfaces. These papers reveal that such irregularities can produce a variety of effects such as wave slowing, stop bands and retroreflection, These results are very important because in practice irregularities are unavoidable.

In Part IV, surface waves on electromagnetic bodies are investigated and a number of electromechanical interaction processes are described. These interactions play an important role in the design of SAW devices such as are used in signal processing. One paper deals with the properties of piezoelectric waves while another discusses current procedures for transducer design.

The main theme of Part $V$ is surface wave propagation in composites with emphasis on acoustic properties of periodic layers, laminated half space and surface and interface waves in a sandwich plate with interface soft layers. The application of these studies is not just limited to waves in structural composites but also to phenomena on the microscopic scale of superlattices.

The concluding part, part IV, deals with wave propagation on curved and fluid-loaded surfaces in viscoelasticity and thermoelasticity. Some of these studies are concerned with surface waves used both in flaw detection in materials and in instruments for evaluating rheological properties of surrounding fluid.

In my opinion the book will be of great value to the reader who wants to acquaint himself with the latest theoretical methods for analysis of surface waves and treatment of a number of novel SAW phenomena. The book also gives valuable suggestions for future research in this rapidly growing field. 
Turbulant Reacting Flows (Topics in Applied Physics, Vol 44)

edited by P A Libby and F A Williams

Springer-Verlag: Berlin-Heldelberg-New York, 1980

$x i+243$ pages, 38 figures, 3 tables ; price : US $\$ 49.60$ (cloth) ; ISBN 3-540-10192-6

The book under review is "Turbulant Reacting Flows" published by SpringerVerlag in 1980 as volume 44, Topics in Applied Physics. It is unusual that a review should appear almost a decade after its appearance! I wonder whether it is timely or it will help those who would like to know whether it is a text worth possessing or pursuing. However I guess it should at least be useful to find out whether a revised edition should be brought out at the present time.

With the developments in the past seven years and more after the appearance of the excellent book the reviewer feels that an augmented edition is overdue. This reviewer feels that specifically two chapters need revision namely Chapter 2 (by A M Mellor and CR Ferguson) dealing with practical problems in turbulant reacting flows and Chapter 6 by PA Libby and F A Williams (the editors of this volume).

Chapters 1, 3, 4 and 5 which deal with the basic physics of turbulant reacting flows and the associated methodology, provide an excellent introduction to the researcher or the beginner entering into the field of turbulant reacting flows. The copious references at the end of these chapters is up to date as of the writing of the book (1980). This should especially be useful to the research worker.

S P VENKATESIIAN Regional Sophisticated Instrumentation Centre, Indian Institute of Technology.

Madras-600 036

Lasers in Photomedicine and Photobiology (Springer Series in Optical Sciences, Vol 22)

(Proceedings of the European Physical Society, Quantum Electronics Division, Conference, Florence, Italy, September 3-6, 1979)

edited by R Pratesi and C A Sacchi

Springer-Verlag: Berlin-Heidelberg-New-York, 1980

235 pages, 108 figures, 20 tables ; price : US $\$ 29.50$; ISBN 3-540-10178-0

The book "Lasers in Photomedicine and Photobiology" of the Springer series in Optical Sciences represents the first critical discussion on the future prospects on non-surgical application of lasers. It is very useful for medical people who are interested in adopting and trying the new discoveries of science to the benefit of mankind. 
The book is divided in six parts, the first of which introduces the subject. The article by A C Giese is of special interest in the introductory part.

In second part, the photodynamic theory of tumors, the effect of Lasers on tumors, injected with Hpd, HP etc. - a contemporary new therapy known as photochemo therapy or photoradiation therapy is discussed. third part.

Photodermatology, effect of lasers on animal and human skin is reported in the

An interesting discussion on phototherapy of Jaundice and Bilirubin is presented in the next part. Some fundamental research like Absorption and fluorescence spectroscopy of biomolecules and their Raman spectra form the part of the chapter five and six. The time resolved spectra of the time of the order of picosecond and subpicosecond region have also been reported in these parts. These studies of laser investigations will increase the knowledge about structures and spectroscopic properties of biomolecules as well as kinetics of various biochemical processes.

In summary, this proceedings of the EPS Quantum Electronic Division, conference, is a useful report and must have inspired many scientists to join this particular line of research.

$$
\begin{array}{r}
\text { R K MUKHERJEE } \\
\text { Department of Solid Stote Physics, } \\
\text { Indian Association for the Cultivation of Science, } \\
\text { Jadavpur, Calcutta-700 } 032
\end{array}
$$

\author{
Structure and Mechanics of Turbulence II (Lecture Notes in Physics, Vol. 76) \\ (Proceedings of Symposium of Turbulence, held at the Technische Universität, Berlin, \\ August 1-5, 1977) \\ edited by $\mathrm{H}$ Fiedler
}

Springer-Verlag : Berlin-Heidelberg-New York, 1978

406 pages, price : US $\$ 17.80$

Tha symposium on Turbulence was held at the Technische Universitat, Berlin during 1st week of August 1977 organised by the Hermann Fottinger Institute Fur Thermo-und Fluiddynamik in joint efforts with the DFVLR-Institut Fur Turbulenzforschung. The symposium consist of survey lectures presented by distinguished experts, research papers and short contributions. The matter which was presented in the symposium is published in two volumes. The present book is the second volume and is entitled as Structure and Mechanisms of Turbulence II edited by $M$ Fiedler. 
One survey lecture and ten research papers in each of the session viz. Passive Scalar Transport, Noise and General Session are presented in this book. At the beginning the survey lecture 'Turbulent Transport: Some General Comments' contains classical background, current progress, research made by author himself and areas of future work followed by an extensive list of references (101 references). Among the rest of the papers in the Passive Scalar Transport session some of them discussed the study about thermal wake produced by heating only on one side of flat plate or by slightly heated cylinder etc. The session also reported the work of the measurement of the turbulence velocity and te.nperature field behind a multi-bore jet block.

The subject Noise covers a wide range of problems of unsteady flow including both deterministic and non-deterministic fluctuations. But the lectures presented in this session were concerned with only limited part of aerodynamic noise generation namely the noise generation of turbulent jets. The important features of supersonic jet and subsonic jet were presented in the survey lecture of this session. The papers about (i) the interaction between a turbulent jet and pure tone sound coming from inside the jet nozzle, (ii) noise from the large scale structure of a jet and (iii) the sound emission from burning puff, from this session are found to be interesting. Using the idea in non-linear hydrodynamic stability, the numerical modelling of the large scale eddies in a turbulent shear flow was described by JTC Liu et al (pp 202-218) to understand the interaction between two separate scales of fluctuations. Further, some of the aerodynamic sound radiation properties of the large scale structure were also described. The effect of shear layer instability on Jet exhaust noise was examined by Moore (pp 245-264) which leads to a new understanding of the jet noise mechanism.

Among the papers in the general session a review article by Saffman (pp 273-306) on the 'Problems and Progress in the Theory of Turbulence' is found to be very much useful. The purpose and motivation of the throretical study of turbulence, the ideas and techniques which had been developed over the past few years, the advantages and shortcomings of different methods are discussed in a simple language. Remaining papers in this session described the theoretical and experimental study of various aspects of turbulent flow.

In conclusion, the mathematical level and arrangement of the papers and articles in this book are quite reasonable. Tha book will certainly be read with interest by Physicist and Engineers in particular from the field of aerodynamics.

P S SALVEKAR 